\title{
Illicit drugs in Canadian municipal wastewater and estimates of community drug use
}

\author{
Chris Metcalfe $^{\mathrm{a}, *}$, Kathryn Tindale ${ }^{\mathrm{a}}$, Hongxia Li $^{\mathrm{a}}$, Angela Rodayan ${ }^{\mathrm{b}}$, Viviane Yargeau ${ }^{\mathrm{b}}$ \\ ${ }^{a}$ Worsfold Water Quality Centre, Trent University, 1600 West Bank Drive Peterborough, ON, K9J 7B8, Canada \\ ${ }^{\mathrm{b}}$ Department of Chemical Engineering, McGill University, 3610 University St., Montreal, QC, H3A 2B2, Canada \\ Cocaine and amphetamines were detected in untreated and treated sewage in the wastewater treatment plants of three Canadian cities, and \\ community consumption patterns estimated from the concentrations of the drugs in untreated wastewater were consistent with estimates of the \\ use of illicit drugs in Canada.
}

\section{A R T I C L E I N F O}

\section{Article history:}

Received 17 March 2010

Received in revised form

28 June 2010

Accepted 1 July 2010

\section{Keywords:}

Illicit drugs

Drugs of abuse

Cocaine

Amphetamines

Wastewater

\begin{abstract}
A B S T R A C T
In this study of wastewater treatment plants in three Canadian cities, selected illicit drugs, including cocaine and its major metabolite, benzoylecgonine (BE), amphetamine, methamphetamine and ecstasy (i.e. MDMA) were detected in untreated wastewater. Cocaine was the most widely used illicit drug at a median level for the 3 cities of 15.7 doses per day per 1000 people. For the other drugs, the median doses per day per 1000 people were 1.8 for amphetamine, 4.5 for methamphetamine and 0.4 for ecstasy. Methamphetamine use was highest in the largest city and cocaine use was lowest in the smallest city. Removal of the illicit drugs by wastewater treatment was generally $>50 \%$, except in a WWTP that uses primary treatment. The community consumption estimate for ecstasy in the present study is far below published estimates of the prevalence of ecstasy use among the Canadian population, which may be due to only occasional use of ecstasy.
\end{abstract}

(c) 2010 Elsevier Ltd. All rights reserved.

\section{Introduction}

In 2008, the United Nations Office on Drugs and Crime (UNODC) reported that an estimated $4.9 \%$ of the world's population aged 15-64 used some type of illicit drug on an annual basis (UNODC, 2008). In Canada alone, it was estimated that 2.3\% of the population aged 15-64 had used cocaine, 1.0\% had used amphetamines and $1.3 \%$ had used ecstasy [UNODC, 2008]. It is difficult to accurately estimate the use of illicit drugs within communities using current indirect survey methods. However, monitoring the levels of drugs of abuse excreted into municipal wastewater has been proposed as a tool for estimating community drug use (Zuccato et al., 2008a). Drug consumption can be estimated from data on the concentrations of the target compounds in untreated wastewater, the flow rates into municipal wastewater treatment plants (WWTPs), the population served by the WWTP, human excretion rates for the target compounds in urine, and estimates of the drug dose. This method

\footnotetext{
* Corresponding author.

E-mail addresses: cmetcalfe@trentu.ca (C. Metcalfe), lihongxia@trentu.ca (H. Li), viviane.yargeau@mcgill.ca (V. Yargeau).
}

has been used to estimate use patterns for drugs of abuse in continental Europe (Zuccato et al., 2005; Castiglioni et al., 2006; Mari et al., 2009; Postigo et al., 2010), in Wales (KasprzykHordern et al., 2009) and in Ireland (Bones et al., 2007). Recently, the concentrations of illicit drugs were determined in wastewater from municipal wastewater treatment plants (WWTPs) in the USA (Chiaia et al., 2008; Bartelt-Hunt et al., 2009; Loganathan et al., 2009), although these studies did not include estimates of community drug consumption. To date, no comparable data on the concentrations of illicit drugs in wastewater have been generated for Canadian municipalities.

The goals of this study were to determine the concentrations of selected illicit drugs in untreated wastewater from three Canadian municipalities that have widely different population sizes and to use these data to estimate community consumption patterns for the drugs. The target compounds included cocaine and its major metabolite, benzoylecgonine, and amphetamine, methamphetamine, MDMA (i.e. ecstasy) and MDA. Wastewater samples were collected throughout the week to monitor for temporal changes in usage. Illicit drugs have been found in rivers and lakes (Postigo et al., 2010; Bartelt-Hunt et al., 2009; Zuccato et al., 2008b; Kasprzyk-Hordern et al., 2008; Castiglioni et al., 2008). Ineffective removal of illicit drugs during wastewater treatment may result in 
contamination of drinking water (Huerta-Fontela et al., 2008), or exposure of fish and other aquatic organisms in surface waters impacted by discharges from WWTPs (Postigo et al., 2010). In this study, the effectiveness of the treatment process for removing illicit drugs was determined by comparing the concentrations of the target compounds in treated and untreated wastewater from the three WWTPs. If there is partitioning or retention of target compounds onto the particulates in wastewater, community drug consumption may be underestimated. The loadings of the target drugs on particulate matter in wastewater were estimated by extracting the particulate material removed by filtration of a bulk wastewater samples (i.e. $>10 \mathrm{~L}$ ).

Wastewater samples were extracted by solid phase extraction (SPE) using MCX cation exchange cartridges. Particulates were extracted using a sonication method developed for this study. The extracts were analyzed by liquid chromatography with tandem mass spectrometry using electrospray ionization (LC-ESI$\mathrm{MS} / \mathrm{MS})$.

\section{Material and methods}

\subsection{Chemicals, reagents and supplies}

Benzoylecgonine, benzoylecgonine-D8, cocaine, cocaine-D3, amphetamine, amphetamine-D8, methamphetamine, methamphetamine-D9, MDMA, MDMA-D5, MDA, and MDA-D5 were purchased from Cerilliant (Round Rock, TX, USA). The deuterated stable isotope standards were used as surrogates for the native compounds to aid in quantification. HPLC grade methanol was purchased from VWR (Mississauga, ON, USA). Ammonium hydroxide and sulfuric acid were obtained from Fisher Scientific (Mississauga, ON, Canada). Oasis MCX cation exchange cartridge $(6 \mathrm{~mL} / 150 \mathrm{mg}$ ) was purchased from Waters Corporation (Milford, MA, USA). Whatman $1.5 \mu \mathrm{m}$ glass microfibre filters were purchased from Fisher Scientific.

\subsection{Sample collection}

Samples of treated and untreated wastewater were obtained from the WWTPs for three municipalities located in eastern Canada. The samples were $24 \mathrm{~h}$ composites, except for one grab sample of untreated effluent that was collected from WWTP3 (Table 1). Table 1 also provides information on the WWTPs, including the populations served, the sampling dates and the wastewater flow rates over the sampling dates, the hydraulic retention times (HRTs) and the wastewater treatment technologies. Note that there were wide differences in the sizes of the populations served by WWTP1 (i.e. 1,600,000), WWTP2 (i.e. 500,000) and WWTP3 (i.e. 75,000). WWTP2 and WWTP3 use secondary treatment with activated sludge, and WWTP1 uses only primary treatment. The wastewater samples were stored at $4{ }^{\circ} \mathrm{C}$ overnight at the WWTPs and then transported to academic laboratories facilities for extraction. The samples were stored in the dark at $4{ }^{\circ} \mathrm{C}$ until they were extracted, which occurred within $48 \mathrm{~h}$ of collection at the WWTP. Extracts were stored in the refrigerator for a maximum of one month before analysis.

\subsection{Extraction of wastewater}

SPE methods were developed to extract the analytes from wastewater by spiking $100 \mathrm{~mL}$ samples of MilliQ water $(n=5)$ with the target compounds ( 50 ng each) and stable isotope surrogates ( $25 \mathrm{ng}$ each). The different methods included extraction with either Oasis HLB or MCX cartridges, the presence or absence of a rinse step prior to cartridge elution, and different volumes of the eluate of ammonium hydroxide in methanol. Recoveries of cocaine and BE were very poor with the HLB cartridge, so the MCX cartridge was selected for further method development. The MCX methods that did not include a rinse step with 5\% methanol generally gave recoveries $>100 \%$; indicating signal enhancement as a result of co-extracted material in the sample matrix. The extraction method that was selected was the most consistent (i.e. coefficient of variation $=3-5 \%$ ) and gave the most accurate recoveries (i.e. $83-101 \%$ ) for the target analytes.

Samples of untreated wastewater samples $(100 \mathrm{~mL})$ and treated wastewater $(200 \mathrm{~mL})$ were vacuum filtered through $1.5 \mu \mathrm{m}$ glass-fibre filters. Each sample was extracted in triplicate. The $\mathrm{pH}$ of the samples was adjusted to 3.0 using $3.5 \mathrm{M}$ sulfuric acid. Each sample was then spiked with $100 \mu \mathrm{L}$ of a $250 \mathrm{ng} / \mathrm{mL}$ surrogate standard mixture and gently mixed. Laboratory blank samples were prepared with MilliQ water. Oasis MCX cartridges were pre-conditioned with $6 \mathrm{~mL}$ of methanol and $10 \mathrm{~mL}$ of $\mathrm{pH} 3.0 \mathrm{HPLC}$ grade water. After pre-conditioning, the wastewater samples were passed through the MCX cartridges at a rate of approximately $10 \mathrm{~mL}$ / min, followed by rinsings (i.e. $10 \mathrm{~mL}$ of pH 3.0 HPLC grade water) from the graduated cylinder that held the sample. The cartridge was dried by aspiration for $1 \mathrm{~min}$, and then rinsed with $3 \mathrm{~mL}$ of $5 \%$ methanol in water. Elution was done immediately following the rinse using a $2 \times 3 \mathrm{ml}$ of $5 \%$ ammonium hydroxide in methanol, followed by a $5 \mathrm{~mL}$ aliquot of $5 \%$ ammonium hydroxide in methanol. Each aliquot of elution solvent was allowed to stand in the SPE for 10 min before being drained into the collection tubing. The samples were evaporated to almost dryness using a vacuum centrifuge apparatus and reconstituted in 50:50 methanol/ water to a final volume of $0.4 \mathrm{~mL}$.

\subsection{Extraction of particulates}

Samples of untreated wastewater were collected from WWTP2 (24 composite) and WWTP3 (grab) and large sample volumes of 11.4 and $12 \mathrm{~L}$, respectively were filtered using multiple $1.5 \mu \mathrm{m}$ glass-fibre filters (i.e. 75 and 80 filters, respectively). All particulates retained on the filters were saved for testing. Aliquots of the filtered wastewater (i.e. $3 \times 100 \mathrm{~mL}$ ) were retained for wastewater analysis. The filters were frozen until the particulates were extracted.

The extraction method was adapted from a method described by Kaleta et al. (2006). The methods were developed by spiking 70 glass-fibre filters with the target compounds ( 50 ng each) and stable isotope surrogates ( 25 ng each). Recoveries of all analytes varied between 70 and $87 \%$. All of the filters with particulate matter were freeze dried, as well as an equal number of clean filters as a lab blank. The freeze dried filters were weighed to determine the dry weight of the particulate material retained on the filters. The filters were then placed in a $250 \mathrm{~mL}$ amber glass jar and a volume of $100 \mu \mathrm{L}$ of the $250 \mathrm{ppb}$ surrogate standard was added. After addition of $100 \mathrm{~mL}$ of $80: 20$ solution of $50 \mathrm{mM}$ formic acid and methanol, the filters were sonicated by immersing the jar in a sonication bath for $15 \mathrm{~min}$. After sonication, the liquid was transferred to a separatory funnel and MilliQ water was added to a final volume of $2000 \mathrm{~mL}$. The filter material was mixed with an additional $100 \mathrm{~mL}$ of the formic acid methanol mixture and was sonicated again for an additional $15 \mathrm{~min}$. The liquid was added to the $2000 \mathrm{~mL}$ in the separatory funnel. The liquid was filtered through

\section{Table 1}

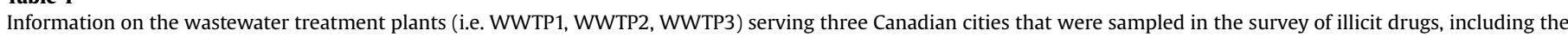

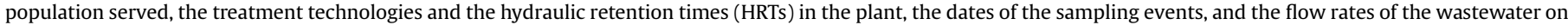
the sampling dates.

\begin{tabular}{|c|c|c|c|c|c|c|c|c|}
\hline Location & Pop $\left(x 10^{6}\right)$ & Treatment technology & HRT (hours) & Sample Name & Sampling Date & Untreated Wastewater & Treated Wastewater & Flow $\left(\mathrm{m}^{3} / \mathrm{d}\right)$ \\
\hline \multirow[t]{8}{*}{ WWTP1 } & 1.6 & Primary & 3 & Monday & $23 / 03 / 09$ & Composite & Composite & 2640 \\
\hline & & & & Tuesday & $24 / 03 / 09$ & Composite & Composite & 2570 \\
\hline & & & & Wednesday & $25 / 03 / 09$ & Composite & Composite & 2560 \\
\hline & & & & Thursday & $26 / 03 / 09$ & Composite & Composite & 2660 \\
\hline & & & & Friday & $27 / 03 / 09$ & Composite & Composite & 3500 \\
\hline & & & & Saturday & $28 / 03 / 09$ & Composite & Composite & 2900 \\
\hline & & & & Sunday & $29 / 03 / 09$ & Composite & Composite & 3810 \\
\hline & & & & Monday 2 & $30 / 03 / 09$ & Composite & Composite & 4600 \\
\hline \multirow[t]{3}{*}{ WWTP2 } & 0.5 & Secondary (activated sludge) & 14 & Week day 1 & $16 / 10 / 08$ & Composite & Composite & 409 \\
\hline & & & & Week day 2 & $21 / 07 / 09$ & Composite $^{a}$ & - & 387 \\
\hline & & & & Weekend 1 & $19 / 10 / 08$ & Composite & Composite & 399 \\
\hline \multirow[t]{3}{*}{ WWTP3 } & 0.075 & Secondary (activated sludge) & 15 & Week day & $09 / 01 / 09$ & Grab $^{\mathrm{a}}$ & - & 41 \\
\hline & & & & Week day & $12 / 08 / 09$ & Composite & Composite & 43 \\
\hline & & & & Weekend & $08 / 08 / 09$ & Composite & Composite & 40 \\
\hline
\end{tabular}

\footnotetext{
${ }^{\text {a }}$ Untreated wastewater collected for analysis of particulates.
} 
a $1.5 \mu \mathrm{m}$ glass-fibre filter, and this filtered solution was extracted by SPE, as described previously for the wastewater samples.

\subsection{Analysis}

Chromatographic separations were carried out with an Agilent 1100 series HPLC system, equipped with a binary pump, an autosampler and an in-line solvent degasser. The analytes were separated chromatographically at room temperature at a flow rate of $0.2 \mathrm{ml} / \mathrm{min}$ on a Genesis C18 column $(150 \mathrm{~mm} \times 3 \mathrm{~mm}, 4 \mu \mathrm{m})$ purchased from Jones Chromatography (Hengoed, U.K.) with a C18 guard column $(4.0 \mathrm{~mm} \times 2.0 \mathrm{~mm})$ purchased from Phenomenex (Torrance, CA). Gradient elution was with a binary mobile phase of $0.1 \%$ formic acid (solution $A$ ) and acetonitrile (solution B). The sample injection volume was $20 \mu \mathrm{L}$. The HPLC was coupled to an MDS/Sciex QTrap mass spectrometer (Concord, ON, Canada) equipped with a Turbolon Spray (i.e ESI) ionization source operated in positive ion mode. Acquisition was performed in selective reaction monitoring (SRM) mode, and the protonated molecular ion of each compound was chosen as the precursor ion. Nitrogen was used as the curtain, nebulizer, auxiliary, and collision gases. The SRM ion transitions monitored for the target analytes and their stable isotope surrogates, and selected optimized operating conditions for LC-MS/MS analysis are listed in Table 2. All data were acquired and processed using Analyst 1.4 software.

Quantification was performed using an external standard method with a five point calibration curve spanning the range of anticipated analyte concentrations in the samples, using a weighted (1/concentration) linear regression. One procedural blank was run for each set of six samples. The stable isotope surrogates (Table 2) were used to compensate for matrix-induced signal suppression and recoveries. The Limits of Detection (LODs) and Limits of Quantitation (LOQs) for wastewater and particulates were determined using a standard additions method, as described at the International Conference on Harmonization of Technical Requirements for the Registration of Pharmaceuticals for Human Use: [www.ich.org/LOB/media/MEDIA417.pdf]. Detection limits were determined by spiking a grab sample of treated wastewater from WWTP3. The LODs and LOQs for the target analytes in wastewater and in particulates are listed in Table 2.

\subsection{Calculation of community drug use}

The method described by Zuccato et al. (2008a) was used to estimate community use of the target drugs. Using the mean $(n=3)$ concentration of the target drug in the samples of untreated wastewater and the daily flow rate in the WWTP, the amount of drug (grams) discharged per day was calculated. This value was then divided by the number of people served by the WWTP to estimate the grams of drug excreted in wastewater per person per day. This value was then normalized to a value of grams per day per 1000 people. Estimates of the fraction of the target drugs excreted in urine provided by Zuccato et al. (2008a), were used to obtain a consumption value for each drug within the community. Cocaine consumption was estimated from the data for its major metabolite, benzylecgonine (BE), so a molar ratio value of 1.05 was applied to compensate for the higher molecular weight of $\mathrm{BE}$ relative to cocaine. In order to determine community drug use (i.e. doses/day/1000 people), the consumption value was divided by an amount considered typical for a single dose of the target drug. The typical doses were $100 \mathrm{mg}$ for intranasal cocaine, $30 \mathrm{mg}$ for ora amphetamine and methamphetamines, and $100 \mathrm{mg}$ for oral ecstasy (Zuccato et al., 2008a).

\section{Results and discussion}

\subsection{Concentrations in wastewater}

All of the target drugs, with the exception of MDA and occasionally methamphetamine and amphetamine were detected at concentrations $>$ LOQs in composite samples of untreated wastewater from the three WWTPs. The data on the frequency of detection at concentrations $>$ LOQ the median concentration and range of concentrations in untreated and treated wastewater are shown in Table 3. Note that the three replicate analyses of each of the wastewater samples were considered to be independent analyses. MDA, which is a metabolite of all amphetamine drugs was detected at concentrations $>$ LOD in 32 of the 40 untreated wastewater samples, but could not be quantified because of the relatively high LOQ of $22 \mathrm{ng} / \mathrm{L}$ for this compound. The maximum concentrations of the other amphetamine compounds in untreated wastewater were all $<70 \mathrm{ng} / \mathrm{L}$ (Table 3 ). It is evident that the concentrations of methamphetamine were higher in the untreated wastewater from WWTP1 relative to the other two sites, but the range of concentrations of amphetamine and MDMA were similar at all three locations. In comparison to data reported for untreated wastewater from WWTPs in the USA (Chiaia et al., 2008), the concentrations of methamphetamine and amphetamine were lower but the levels of MDMA (i.e. ecstasy) were comparable to the present study. In Europe, methamphetamine is generally present at lower concentrations (i.e. $<10 \mathrm{ng} / \mathrm{L}$ ) in untreated wastewater, but the concentrations of MDMA and amphetamine are comparable to the levels reported in the present study (Zuccato et al., 2005; Castiglioni et al., 2006; Postigo et al., 2010).

The concentrations of BE and cocaine varied between 287 and $2624 \mathrm{ng} / \mathrm{L}$ and 209 and $823 \mathrm{ng} / \mathrm{L}$, respectively in samples of the untreated wastewater (Table 3 ). These concentrations are very similar to the range of concentrations of these compounds detected in untreated wastewater from WWTPs in the USA (Chiaia et al., 2008) and in Europe (Zuccato et al., 2005; Castiglioni et al., 2006; Mari et al., 2009; Postigo et al., 2010). Fig. 1 illustrates the mean concentrations of cocaine and its major metabolite, BE in wastewater samples collected at the three WWTPs. The ratios between mean $\mathrm{BE}$ concentrations and mean cocaine concentrations in the wastewater were highly variable; between 0.88 and 3.20 . The lowest ratios were observed in samples collected on week days at WWTP1 (Fig. 1). These ratios are markedly different from the ratios observed in untreated wastewater from cities in continental Europe (Zuccato et al., 2008a, 2005; Castiglioni et al., 2006; Mari et al., 2009; Postigo et al., 2010) and in the USA (Chiaia et al., 2008),

Table 2

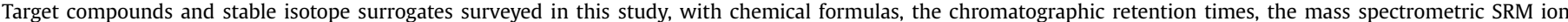

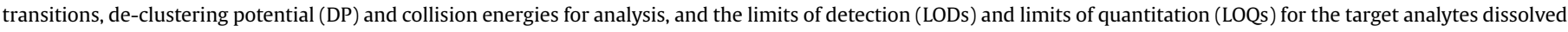
in wastewater (ng/L) and adsorbed to particulates ( $\mathrm{ng} / \mathrm{g}$ dry weight).

\begin{tabular}{|c|c|c|c|c|c|c|c|}
\hline Compound & Chemical Formula & Retention Time & SRM Transition & $\mathrm{DP}(\mathrm{V})$ & Collision Energy $(\mathrm{eV})$ & Wastewater LOD, LOQ (ng/L) & Particulate LOD, LOQ (ng/g dw) \\
\hline Cocaine & $\mathrm{C}_{17} \mathrm{H}_{21} \mathrm{NO}_{4}$ & 12.8 & $304 \rightarrow 182$ & 51 & 26 & 6,20 & $0.05,0.16$ \\
\hline Cocaine-d3 & & 12.8 & $307 \rightarrow 185$ & 52 & 25 & - & - \\
\hline Benzoylecgonine (BE) & $\mathrm{C}_{16} \mathrm{H}_{19} \mathrm{NO}_{4}$ & 11.9 & $290 \rightarrow 168$ & 63 & 24 & 6,20 & $0.05,0.16$ \\
\hline Benzoylecgonine-d8 & & 11.8 & $298 \rightarrow 171$ & 55 & 27 & - & - \\
\hline Amphetamine & $\mathrm{C}_{9} \mathrm{H}_{13} \mathrm{~N}$ & 10.6 & $136 \rightarrow 91$ & 46 & 25 & 1,3 & $0.01,0.03$ \\
\hline Amphetamine-d8 & & 10.5 & $144 \rightarrow 99$ & 51 & 25 & - & - \\
\hline Methamphetamine & $\mathrm{C}_{10} \mathrm{H}_{15} \mathrm{~N}$ & 11.1 & $150 \rightarrow 91$ & 48 & 25 & 2,7 & $0.01,0.03$ \\
\hline Methamphetamine-d9 & & 11.0 & $159 \rightarrow 93$ & 48 & 25 & - & - \\
\hline MDA & $\mathrm{C}_{10} \mathrm{H}_{13} \mathrm{NO}_{2}$ & 10.9 & $180 \rightarrow 105$ & 44 & 30 & 7,22 & $0.04,0.12$ \\
\hline MDA-d5 & & 11.2 & $185 \rightarrow 110$ & 44 & 33 & - & - \\
\hline MDMA & $\mathrm{C}_{11} \mathrm{H}_{15} \mathrm{NO}_{2}$ & 11.3 & $194 \rightarrow 105$ & 44 & 33 & 3,9 & $0.02,0.06$ \\
\hline MDMA-d5 & & 11.2 & $199 \rightarrow 107$ & 44 & 33 & - & - \\
\hline
\end{tabular}


Table 3

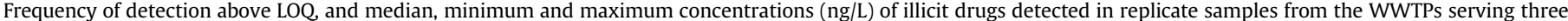

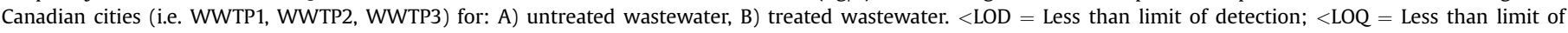
quantitation.

\begin{tabular}{|c|c|c|c|c|c|c|c|c|c|}
\hline \multicolumn{10}{|c|}{ A) Untreated wastewater } \\
\hline \multirow[t]{2}{*}{ Compound } & \multicolumn{3}{|l|}{ WWTP1 } & \multicolumn{3}{|l|}{ WWTP2 } & \multicolumn{3}{|l|}{ WWTP3 } \\
\hline & $\begin{array}{l}\text { Freq }(\%) \\
n=24\end{array}$ & $\begin{array}{l}\text { Median Conc. } \\
(\mathrm{ng} / \mathrm{L})\end{array}$ & $\begin{array}{l}\text { Range of Conc. } \\
\text { (ng/L) }\end{array}$ & $\begin{array}{l}\text { Freq (\%) } \\
n=9\end{array}$ & $\begin{array}{l}\text { Median Conc. } \\
(\mathrm{ng} / \mathrm{L})\end{array}$ & $\begin{array}{l}\text { Range of Conc. } \\
(\mathrm{ng} / \mathrm{L})\end{array}$ & $\begin{array}{l}\text { Freq }(\%) \\
n=9\end{array}$ & $\begin{array}{l}\text { Median Conc. } \\
\text { (ng/L) }\end{array}$ & $\begin{array}{l}\text { Range of Conc. } \\
\text { (ng/L) }\end{array}$ \\
\hline MDA & 0 & - & $<\mathrm{LOQ}$ & 0 & - & $<$ LOQ & 0 & - & $<\mathrm{LOQ}$ \\
\hline Amphetamine & 87.5 & 14 & $<$ LOQ-25 & 100 & 14 & $12-15$ & 100 & 18 & $15-22$ \\
\hline Methamphetamine & 100 & 44 & $21-65$ & 100 & 18 & $16-19$ & 67 & 3 & $<$ LOQ-5 \\
\hline MDMA & 100 & 27 & $10-35$ & 100 & 24 & $9-35$ & 100 & 15 & $9-20$ \\
\hline Cocaine & 100 & 527 & $209-823$ & 100 & 623 & $475-776$ & 100 & 567 & $487-656$ \\
\hline $\mathrm{BE}$ & 100 & 643 & $287-1488$ & 100 & 893 & $628-2624$ & 100 & 612 & $588-722$ \\
\hline \multicolumn{10}{|c|}{ B) Treated wastewater } \\
\hline \multirow[t]{2}{*}{ Compound } & \multicolumn{3}{|l|}{ WWTP1 } & \multicolumn{3}{|l|}{ WWTP2 } & \multicolumn{3}{|l|}{ WWTP3 } \\
\hline & $\begin{array}{l}\text { Freq }(\%) \\
n=24\end{array}$ & $\begin{array}{l}\text { Median Conc. } \\
(\mathrm{ng} / \mathrm{L})\end{array}$ & $\begin{array}{l}\text { Range of Conc. } \\
(\mathrm{ng} / \mathrm{L})\end{array}$ & $\begin{array}{l}\text { Freq }(\%) \\
n=6\end{array}$ & $\begin{array}{l}\text { Median Conc. } \\
(\mathrm{ng} / \mathrm{L})\end{array}$ & $\begin{array}{l}\text { Range of Conc. } \\
(\mathrm{ng} / \mathrm{L})\end{array}$ & $\begin{array}{l}\text { Freq }(\%) \\
n=6\end{array}$ & $\begin{array}{l}\text { Median Conc. } \\
\text { (ng/L) }\end{array}$ & $\begin{array}{l}\text { Range of Conc. } \\
\text { (ng/L) }\end{array}$ \\
\hline MDA & 0 & - & $<\mathrm{LOQ}$ & 0 & - & $<$ LOQ & 0 & - & $<$ LOQ \\
\hline Amphetamine & 37.5 & 7 & $<$ LOD-14 & 0 & - & $<\mathrm{LOQ}$ & 0 & - & $<$ LOD \\
\hline Methamphetamine & 100 & 56 & $17-95$ & 100 & 8 & $7-9$ & 50 & 2 & $<$ LOD-3 \\
\hline MDMA & 87.5 & 25 & $<$ LOD-32 & 50 & 9 & $<$ LOQ-13 & 50 & 7 & $<$ LOQ-9 \\
\hline Cocaine & 100 & 348 & $122-530$ & 50 & 17 & $<$ LOQ-39 & 50 & 25 & $<$ LOQ-34 \\
\hline $\mathrm{BE}$ & 100 & 452 & $142-775$ & 100 & 90 & $62-117$ & 100 & 166 & $116-214$ \\
\hline
\end{tabular}

where cocaine concentrations are typically $<50 \%$ of the concentrations of BE. About $45 \%$ of the initial dose of cocaine is excreted in urine as BE, while about $9 \%$ is excreted as the parent compound (Kasprzyk-Hordern et al., 2009), for an expected value of 5 for the $\mathrm{BE}$ to cocaine ratio, assuming that there is no degradation of either compound in the sewage. In those instances where the ratios between $\mathrm{BE}$ and cocaine were low (Fig. 1), the proportions may have been skewed by accidental or deliberate disposal of raw cocaine into the municipal sewage system. This hypothesis requires further study, but it interesting to note that even commercial drug manufacturers release large amounts of product into municipal wastewater (Phillips et al., 2010). Higher concentrations of cocaine relative to BE were also noted in wastewater from a WWTP in Ireland (Bones et al., 2007). The authors of this study speculated that this may be due to removal of $\mathrm{BE}$ on particulates during sample filtration, since $\mathrm{BE}$ is present as a neutral species and cocaine is present as a more water soluble cation at circum-neutral $\mathrm{pH}$.

Table 4 shows the amounts and proportions relative to wastewater of the target compounds retained on the particulates filtered from bulk samples of untreated wastewater collected at WWTP2 and WWTP3. These data show that the proportions of the target compounds retained on the filter were relatively low when calculated on a ng/L or a ng/g dry weight basis, with the possible exception of MDA, which was present at quantifiable concentrations on the

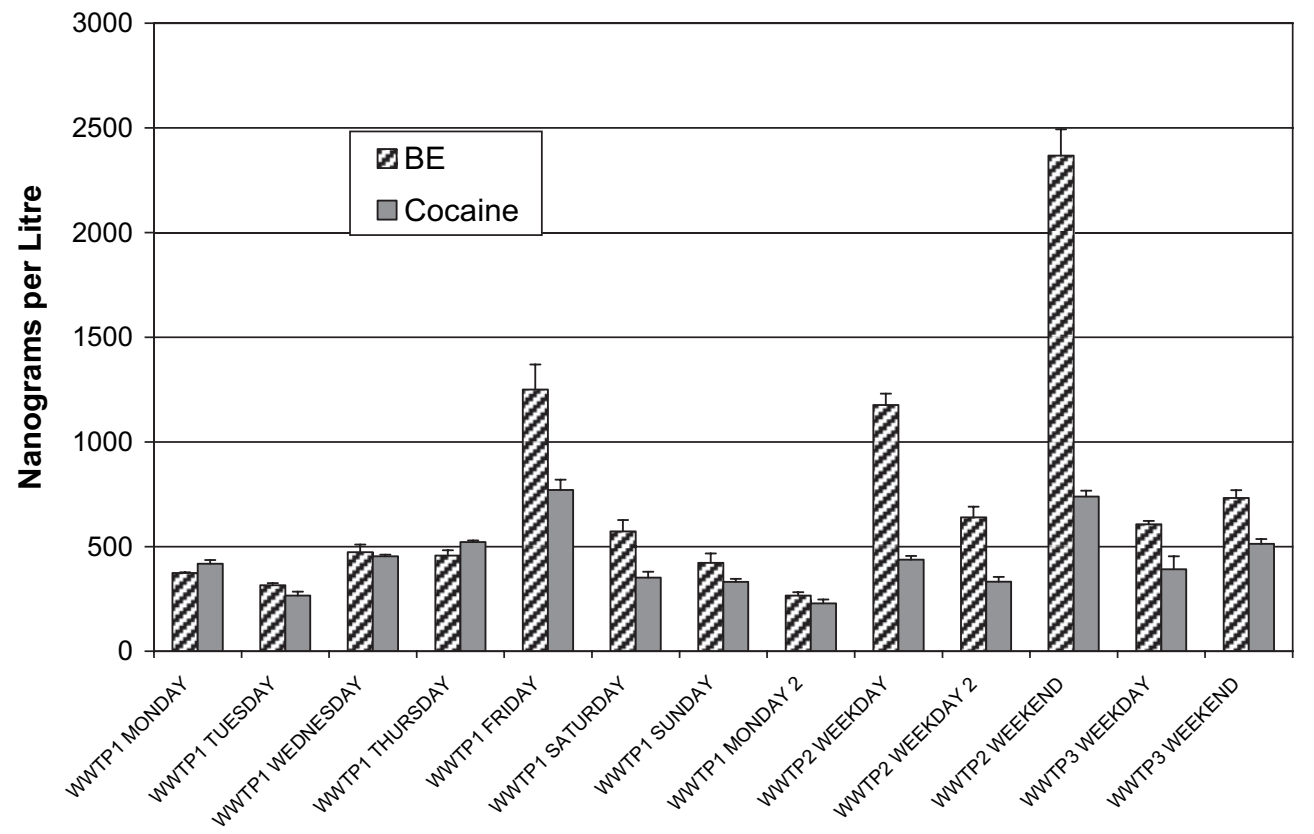

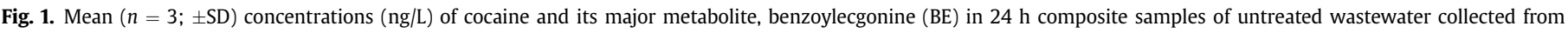
WWTPs that serve three Canadian communities. 
Table 4

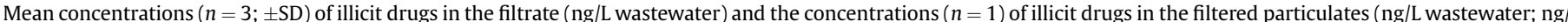

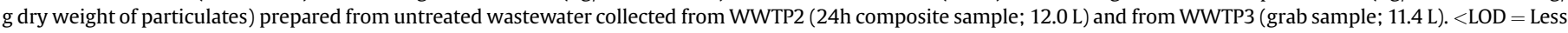

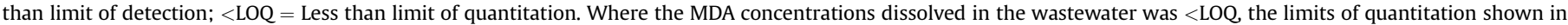
brackets were used to estimate the proportions (\%) adsorbed to the particulates.

\begin{tabular}{|c|c|c|c|c|c|c|}
\hline & \multicolumn{3}{|l|}{ WWP2 } & \multicolumn{3}{|l|}{ WWTP3 } \\
\hline & $\begin{array}{l}\text { Mean }(n=3) \text { concentration } \\
\text { in wastewater filtrate }(n g / L)\end{array}$ & $\begin{array}{l}\text { Concentration in filtered } \\
\text { particulates (ng/L; ng/g } \\
\mathrm{dw})\end{array}$ & $\begin{array}{l}\text { Proportion on } \\
\text { Particulates (\%) }\end{array}$ & $\begin{array}{l}\text { Mean }(n=3) \text { concentration } \\
\text { in wastewater filtrate }(n g / L)\end{array}$ & $\begin{array}{l}\text { Concentration in filtered } \\
\text { particulates (ng/L; ng/g } \\
\mathrm{dw} \text { ) }\end{array}$ & $\begin{array}{l}\text { Proportion on } \\
\text { Particulates (\%) }\end{array}$ \\
\hline Amphetamine & $15 \pm 1$ & $<$ LOD & - & $11 \pm 1$ & $<$ LOD & - \\
\hline Methamphetamine & $17 \pm 1$ & $<1 ; 0.14$ & 5.9 & $17 \pm 3$ & $<1 ; 0.31$ & 6.3 \\
\hline MDA & $<$ LOQ & $<$ LOD & - & $<$ LOQ (22) & $4 ; 2.73$ & 18.0 \\
\hline MDMA & $13 \pm 4$ & $<1 ; 0.07$ & 3.3 & $13 \pm 1$ & $<$ LOD & - \\
\hline Cocaine & $733 \pm 52$ & $17 ; 5.14$ & 2.3 & $639 \pm 18$ & $24 ; 16.90$ & 2.7 \\
\hline $\mathrm{BE}$ & $639 \pm 12$ & $14 ; 4.67$ & 2.2 & $433 \pm 16$ & $14 ; 9.60$ & 3.0 \\
\hline
\end{tabular}

particulates but detected at $<$ LOQ in the wastewater at WWTP3 (Table 4). Therefore, it is unlikely that the filtration of wastewater prior to SPE extraction will result in significant underestimates of the concentrations of illicit drugs in wastewater. There did not appear to be any bias towards retention of $\mathrm{BE}$ on the filter that could result in detection of higher amounts of cocaine relative to $\mathrm{BE}$ in the filtered wastewater samples, as was suggested previously (Bones et al., 2007).

Samples (24 h composite) of final treated wastewater were analyzed for comparison to the untreated wastewater data to determine the percent removals of the target compounds within the three WWTPs. Fig. 2 shows that cocaine, BE and the amphetamine drugs were generally removed in WWTP2 and WWTP3 with efficiencies $>50 \%$. Note that MDA was not included in these analyses because concentrations were generally $<$ LOQs in untreated wastewater. In WWTP1, there was a much lower percent removal of all target analytes, with the possible exception of amphetamine (Fig. 2), which can be attributed to the primary treatment technologies used in this plant. Because of WWTP1, the maximum concentrations of the target compounds detected in the present study in treated wastewater (Table 3 ) were generally higher or close to the maximum concentrations reported for treated wastewater in WWTPs from Europe (Zuccato et al., 2005; Postigo et al., 2010).

\subsection{Community drug usage}

Fig. 3 illustrates the estimates of community use of the illicit drugs within the Canadian cities served by the three WWTPs. Calculations from the data for BE indicate that cocaine is the most commonly used illicit drug in Canadian cities, which is consistent with consumption estimates for other cities in continental Europe, Wales and Ireland (Zuccato et al., 2008a, 2005; Castiglioni et al., 2006; Postigo et al., 2010; Kasprzyk-Hordern et al., 2009; Bones et al., 2007). The estimates for the number of doses of cocaine varied widely from a low of 8.1 per day per 1000 people on a week day in the city served by WWTP3 to a high of 56.7 per day per 1000 people on a Friday in the city served by WWTP1 (Fig. 3), with

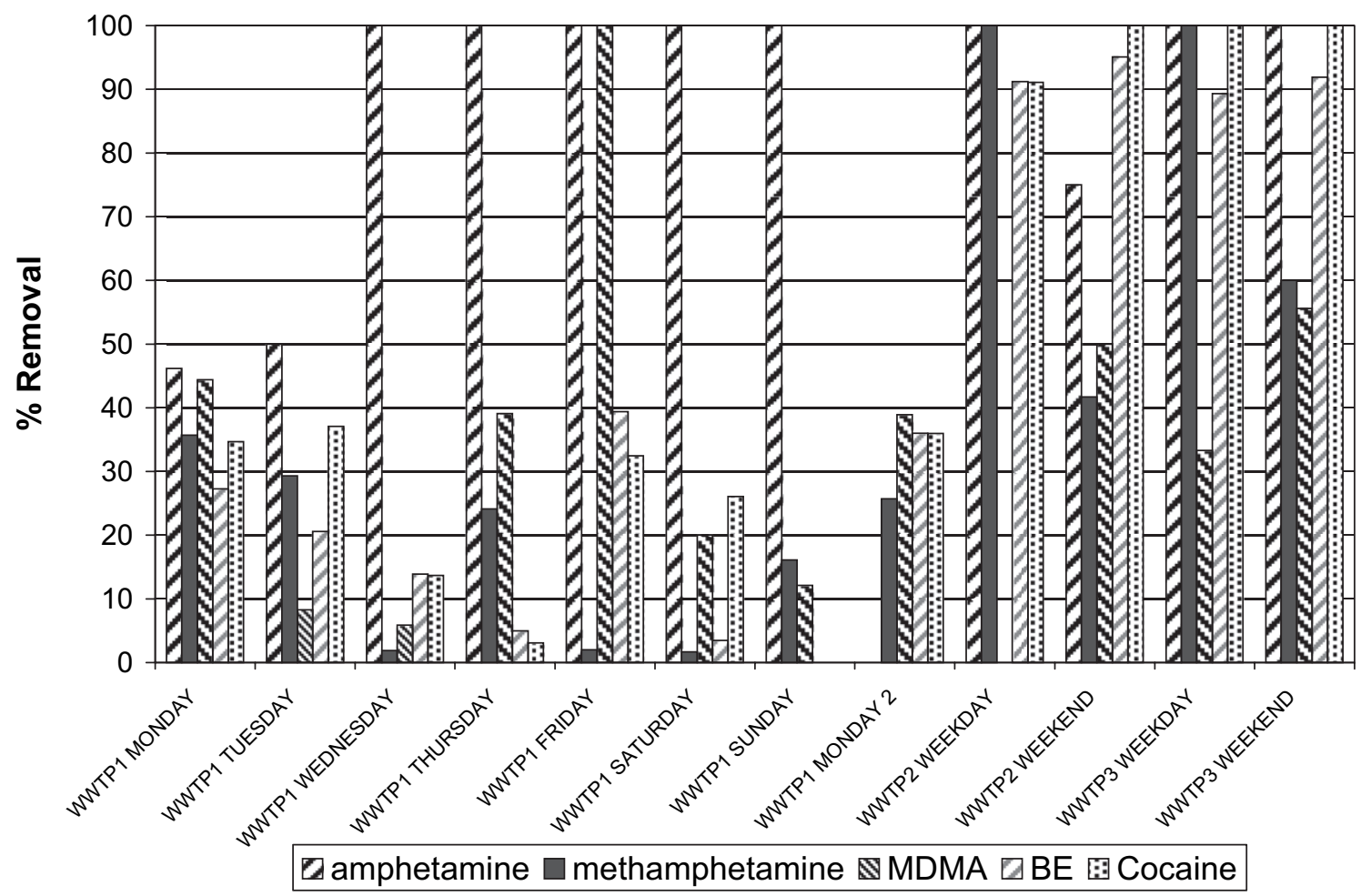

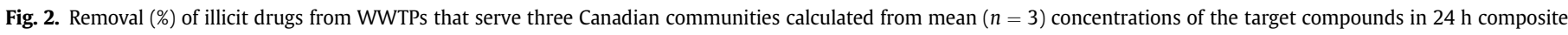

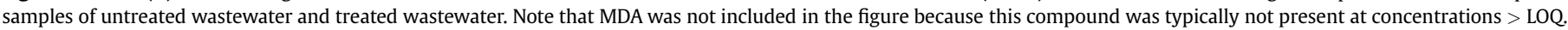




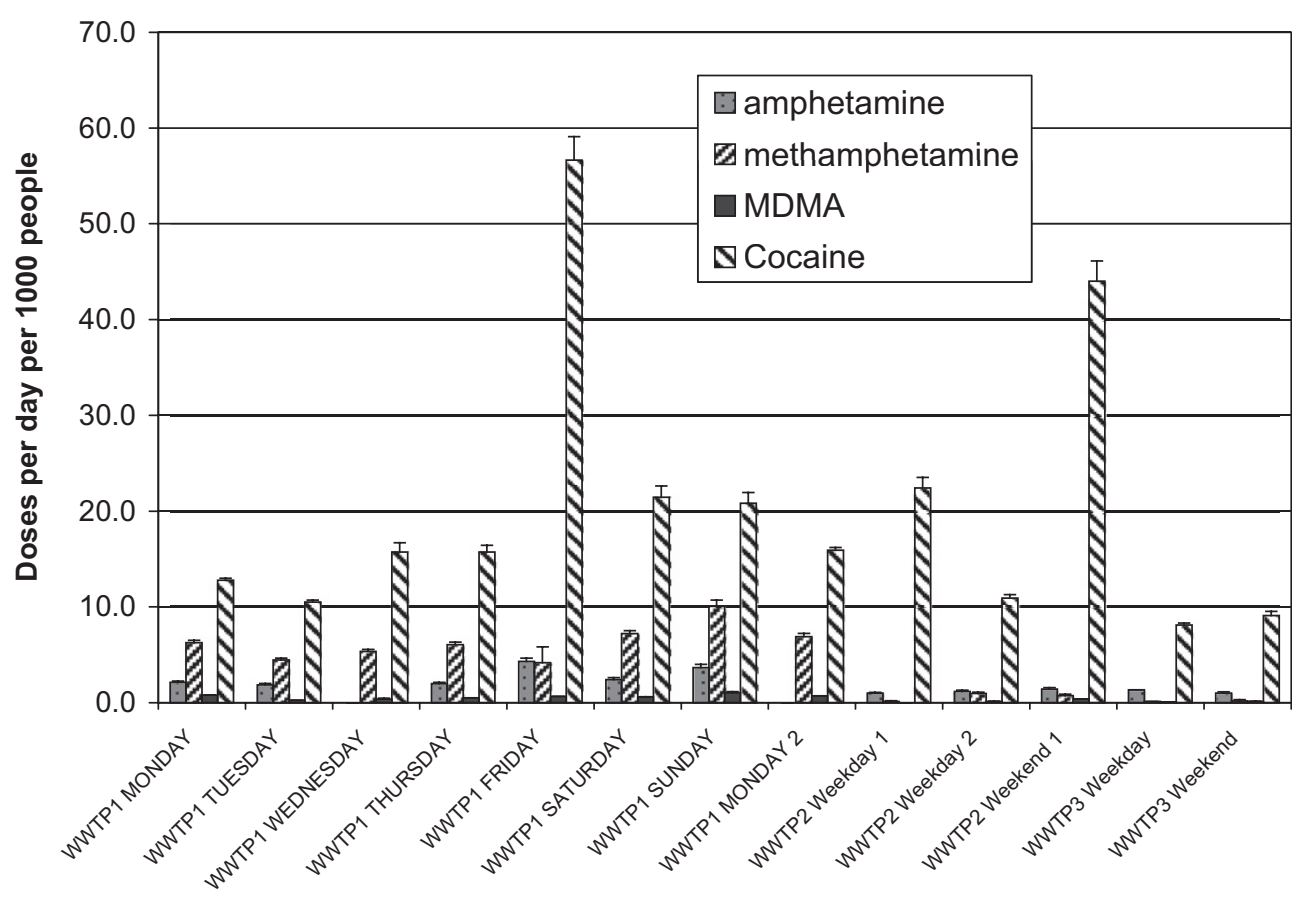

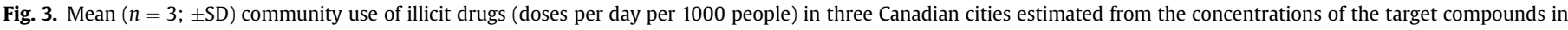

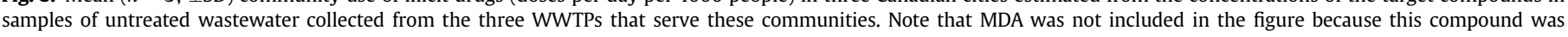
typically not present at concentrations $>$ LOQ.

a median value of 15.7 doses of cocaine per day per 1000 people. It appears that cocaine consumption increased slightly on weekends in the communities served by all three WWTPs (Fig. 3). Note that these estimates of cocaine use were derived from data on $\mathrm{BE}$ concentrations in wastewater, which do not take into account any cocaine that is present in wastewater as a result of direct disposal into the sewage system.

Consumption of amphetamines, and in particular, methamphetamine was greatest in the city served by WWTP1 (Fig. 3). For instance, use of methamphetamine in the city served by WWTP1 varied over the sampling period from 4.2 to 10.1 doses per day per 1000 people, but this drug was consumed at $<1$ dose per day per 1000 people in the cities served by WWTP2 and WWTP3. Estimated weekend use (i.e. Friday to Monday) of amphetamine, methamphetamine and MDMA also appeared to be higher than the estimated week day use in the city served by WWTP1 (Fig. 3). Amphetamine consumption can be from use of prescription drugs, in addition to abuse of illegal drugs. The median values for consumption of amphetamines for all three Canadian cities were 1.8, 4.5 and 0.4 doses per day per 1000 people for amphetamine, methamphetamine and MDMA, respectively.

These community consumption estimates appear to show that cocaine use is lowest in the small city served by WWTP3, and that methamphetamine use is highest in the large city served by WWTP1. These data could be interpreted as evidence that there is greater drug use in large Canadian cities; possibly because of greater access to the distribution networks for illicit drugs. However, it must be noted that the population demographics and socioeconomic conditions vary across the three Canadian cities. The city served by WWTP1 is a large urban center with considerable cultural diversity and a wide age distribution. The small city served by WWTP3 has an economy that is primarily service based, an older age demographic and relatively low cultural diversity. Therefore, any interpretation of drug use patterns among cities in Canada, and elsewhere will require an evaluation of the demographic, cultural, economic, criminal and societal conditions within these urban centers.

The World Drug Report published by UNODC (2008) provided estimates of the prevalence of drug use for the Canadian population aged $15-64$ of $2.3 \%$ for cocaine, $1.0 \%$ for amphetamine and methamphetamine combined, and $1.3 \%$ for ecstasy (i.e. MDMA). The median estimates of community drug use of $0.18 \%$ for amphetamine, $0.45 \%$ for methamphetamine and $1.57 \%$ for cocaine determined in the present study are consistent with the World Drug Report data on the prevalence of drug use in Canada (UNODC, 2008), although these estimates are not directly comparable because wastewater comes from the total population and not just the age 15-64 demographic. In addition, prevalence data may overestimate the use of drugs that are used occasionally by many (e.g. ecstasy), rather than used steadily by a few (Zuccato et al., 2008a). The community consumption estimate of $0.04 \%$ generated for MDMA (i.e. ecstasy) in the present study is far below the WDR prevalence estimate of $1.3 \%$ in Canada. If data from the present study are accurate, the use of MDMA in Canada may actually be closer to the lower rate of ecstasy use that has been estimated for many countries in continental Europe (UNODC, 2008). More studies are required to confirm this conclusion.

\section{Conclusions}

This survey of the WWTPs from a limited number of Canadian cities demonstrates that analytical data for untreated wastewater can be used to estimate community consumption of illicit drugs. The data in the present study are the first published estimates generated using this tool for illicit drug use in North American cities. Community drug use estimates for the Canadian cities are generally consistent with the estimates of drug use that have been generated for European cities using this method, although methamphetamine use appears to be higher in Canada. In comparison to studies conducted in Europe and the USA, the concentrations of 
cocaine were sometimes high relative to the concentrations of the metabolite, BE in untreated wastewater from the three Canadian cities. Further investigation is required to confirm these observations, and if confirmed, to evaluate whether this can be attributed to accidental or deliberate disposal of cocaine directly into sewers. Further work is required to determine whether other illicit drugs, such as opioids, cannibinoids, PCP and LSD, and their metabolites are present in the wastewater from Canadian cities, as has been shown for cities in both Europe and the USA (Postigo et al., 2010; Zuccato et al., 2008a; Chiaia et al., 2008). It would also be useful to determine the levels of these compounds in surface waters in Canada that are impacted by discharges of municipal wastewater to evaluate the potential for biological effects in exposed aquatic organisms or for contamination of sources of drinking water.

\section{Acknowledgements}

We gratefully acknowledge the assistance of the staff of the wastewater treatment plants for collecting the samples. We thank Maria Figueroa Cano for her assistance in preparing many of the samples for analysis. This work was supported by the Natural Sciences and Engineering Research Council (NSERC) of Canada through a Discovery Grant awarded to Dr. Viviane Yargeau and through a Strategic Grants for a project coordinated by Dr. Joanna Wilson (Principal Investigator) of McMaster University, Canada.

\section{References}

Bartelt-Hunt, S., Snow, D.D., Damon, T., Shockley, J., Hoagland, K., 2009. The occurrence of illicit and therapeutic pharmaceuticals in wastewater effluent and surface waters in Nebraska. Environ. Pollut. 157, 786-791.

Bones, J., Thomas, K.V., Paull, B., 2007. Using environmental analytical data to estimate levels of community consumption of illicit drugs and abused pharmaceuticals. J. Environ. Monit. 9, 701-707.

Castiglioni, S., Zuccato, E., Chiabrando, C., Fanelli, R., Bagnati, R., 2008. Mass spectrometric analysis of illicit drugs in wastewater and surface water. Mass Spectrom. Rev. 27, 378-394.
Castiglioni, S., Zuccato, E., Crisci, E., Chiabrando, C., Fanelli, R., Bagnati, R., 2006. Identification and measurement of illicit drugs and their metabolites in urban wastewater by liquid chromatography-tandem mass spectrometry. Anal. Chem. 78, 8421-8429.

Chiaia, A.C., Banta-Green, C., Field, J., 2008. Eliminating solid phase extraction with large volume injection LC/MS/MS: analysis of illicit and legal drugs and human urine indicators in US wastewaters. Environ. Sci. Technol. 42, 8841-8848.

Huerta-Fontela, M., Galceran, M.T., Ventura, F., 2008. Stimulatory drugs of abuse in surface waters and their removal in a conventional drinking water treatment plant. Environ. Sci. Technol. 42, 6809-6816.

Kaleta, A., Ferdig, M., Buchberger, W., 2006. Semiquantitative determination of residues of amphetamine in sewage sludge samples. J. Sep. Sci. 29, 1662-1666.

Kasprzyk-Hordern, B., Dinsdale, R.M., Guwy, A.J., 2009. Illicit drugs and pharmaceuticals in the environment - forensic applications of environmental data. Part 1: estimation of the usage of drugs in local communities. Environ. Pollut. 157, 1773-1777.

Kasprzyk-Hordern, B., Dinsdale, R.M., Guwy, A.J., 2008. The occurrence of pharmaceuticals, personal care products, endocrine disruptors and illicit drugs in surface water in South Wales, UK. Water Res. 42, 3498-3518.

Loganathan, B., Phillips, M., Mowery, H., Jones-Lepp, T.L., 2009. Contamination profiles and mass loadings of macrolide antibiotics and illicit drugs from a small urban wastewater treatment plant. Chemosphere 75, 70-77.

Mari, F., Politi, L., Biggeri, A., Accetta, G., Trignano, C., Di Padua, M., Bertol, E., 2009. Cocaine and heroin in waste water plants: a 1-year study in the city of Florence, Italy. Forensic Sci. Int. 189, 88-92.

Phillips, P.J., Smith, S.G., Kolpin, D.W., Zaugg, S.D., Buxton, H.T., Furlong, E.T., Esposito, K., Stinson, B., 2010. Pharmaceutical formulations facilities as sources of opioids and other pharmaceuticals to wastewater treatment plant effluents. Environ. Sci. Technol. 44, 4910-4916.

Postigo, C. de Alda, M.J.L., Barceló, D., 2010. Drugs of abuse and their metabolites in the Ebro River basin: occurrence in sewage and surface water, sewage treatment plants removal efficiency, and collective drug usage estimation. Environ. Int. 36, 75-84.

UNODC, 2008. World Drug Report 2008. United Nations Office on Drugs and Crime, Vienna International Centre, Vienna, Austria. http://www.unodc.org/documents/ wdr/WDR_2008/WDR_2008_eng_web.pdf 310pp.

Zuccato, E., Chiabrando, C., Castigliano, S., Bagnati, R., Fanelli, R., 2008a. Estimating community drug abuse by wastewater analysis. Environ. Health Perspect. 116, 1027-1032.

Zuccato, E., Castiglioni, S., Bagnati, R., Chiabrando, C., Grassi, P., Fanelli, R., 2008b. Illicit drugs, a novel group of environmental contaminants. Water Res. 42, 961-968.

Zuccato, E., Chiabrando, C., Castiglioni, S., Calamari, D., Bagnati, R., Schiarea, S., Fanelli, R., 2005. Cocaine in surface water: a new evidence based tool to monitor community drug abuse. Environ. Health: A Global Access Science Source 4, 1-14. 